\title{
VITAMIN D STATUS OF ADULTS WITH GROWTH HORMONE DEFICIENCY
}

Ivayla Uzunova', Georgi Kirilov ${ }^{1}$, Sabina Zacharieva ${ }^{1}$, Krassimir Kalinov

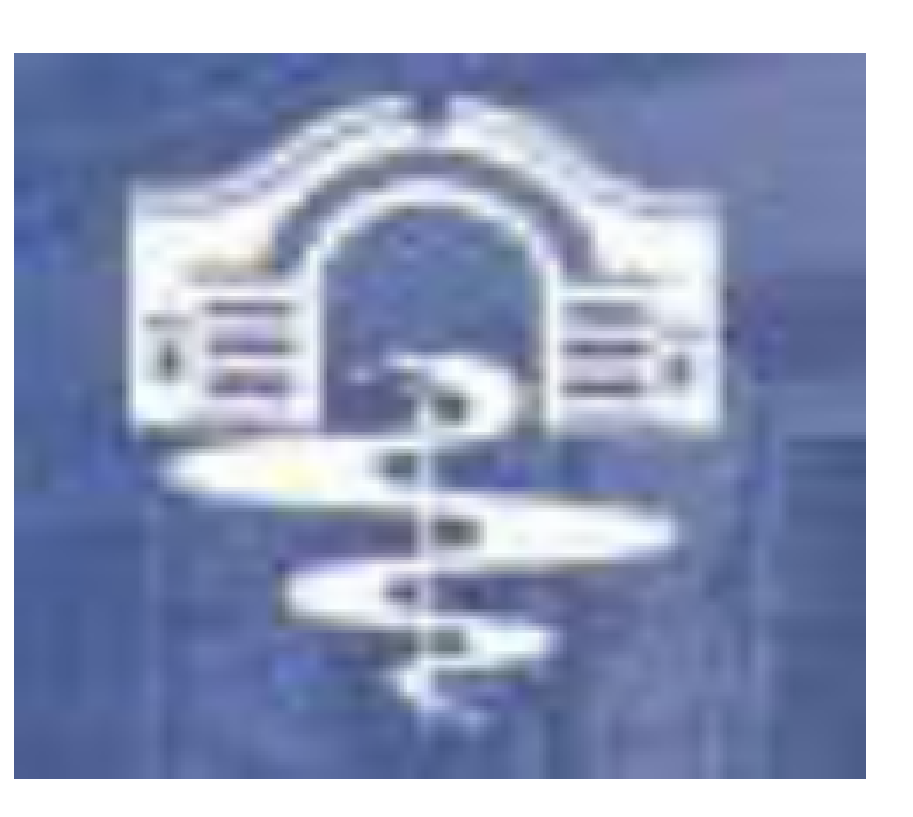

1Department of Hypothalamic, Pituitary, Adrenal and Gonadal Diseases

Clinical Centre of Endocrinology and Gerontology

Medical University - Sofia

${ }^{2}$ New Bulgarian University, Sofia, Bulgaria

2 Zdrave Str., Sofia, Bulgaria; e-mail: dr_uzunova@yahoo.com

Vitamin $D$ and growth hormone/insulin-like growth factor-1 (GH/IGF-1) axis are reported to interplay at both endocrine and paracrine levels, with a positive correlation between IGF-1 and 25-hydroxyvitamin $D(25(\mathrm{OH}) \mathrm{D})$ in healthy subjects. Hyposomatotropism is among the conditions predisposing to lower vitamin $D$ status due to several direct and indirect mechanisms.

\section{Aim of the research}

The aim of the study was to evaluate vitamin $D$ status in a representative sample of adults with GH deficiency (GHD) and to investigate the association between serum 25(OH)D and age, gender and onset of hyposomatotropism.

\section{Patients and methods}

This cross-sectional study included 129 adult patients (70 males, aged 42.1 16.6 years) diagnosed with GHD [childhood-onset GHD (COGHD): $n=54]$ in the Clinical Centre of Endocrinology in Sofia, Bulgaria (latitude $41^{\circ}-44^{\circ} \mathrm{N}$ ). Total serum 25(OH)D was the vitamin $D$ metabolite used to assess vitamin $D$ status and was measured by electro-chemiluminescence binding assay (COBAS, Roche Diagnostics International Ltd.; analytical sensitivity - $4.01 \mathrm{ng} / \mathrm{ml}$; within-run and intermediate precisions - $\leq 6.5 \%$ and $\leq 11.5 \%$, respectively) which employs vitamin $D$ binding protein to capture both 25-hydroxyvitamin D3 and D2. Vitamin D status and GHD were defined according to the Endocrine Society Clinical Practice Guideline recommendations. Statistical analysis was performed using SPSS for Windows, version 23.0.

\section{Results}

Serum 25(OH)D levels were evaluated in 70 men and 59 women with hyposomatotropism, with men being 8 years younger in their mean age $(p=0.006)$. The two study subgroups divided in accordance with the onset of the GHD also differed significantly in their mean age - the 54 subjects with COGHD were almost twice younger than AOGHD patients $(p<0.0001)$. (Table 1$)$

\begin{tabular}{|l|c|c|c|c|c|}
\hline & All & Men & Women & COGHD & AOGHD \\
\hline Number (\%) & $129(100 \%)$ & $70(54.3 \%)$ & $59(45.7 \%)$ & $54(41.9 \%)$ & $75(58.1 \%)$ \\
\hline Age min - max (years) & $18-82$ & $18-77$ & $18-82$ & $18-58$ & $20-82$ \\
\hline Mean age SD (years) & 42.116 .6 & $38.516 .4^{*}$ & $46.516 .1^{*}$ & $29.412 .1^{* *}$ & $51.312 .9^{* *}$ \\
\hline
\end{tabular}

\section{Table 1. Descriptive characteristics of the study population}

${ }^{*} p<0.05$ mean age of men vs. women

${ }^{* *} p<0.05$ mean age of COGHD patients vs. AOGHD patients
Only $6.2 \%(n=8)$ of our study participants had adequate vitamin $D$ status with serum $25(\mathrm{OH}) \mathrm{D}$ concentrations of $30 \mathrm{ng} / \mathrm{ml}$ or higher The predominant part of the study cohort was diagnosed with vitamin $D$ deficiency - 102 subjects $(\mathbf{7 9 . 1 \% )}$; the remaining $14.7 \%$ $(n=19)$ of the patients were with vitamin $D$ insufficiency (serum 25(OH)D between 20 and $29.9 \mathrm{ng} / \mathrm{ml}$ ). (Table 2)

\begin{tabular}{|l|c|c|c|c|c|}
\hline $\mathbf{2 5}(\mathbf{O H}) D(\mathbf{n g} / \mathbf{m l})$ & All & Men & Women & COGHD & AOGHD \\
\hline Min - Max & $3.9-49.9$ & $6.8-35.6$ & $3.9-49.9$ & $5.4-35.6$ & $3.9-49.9$ \\
\hline Mean \pm SD & $15.1 \pm 7.6$ & $15.7 \pm 7.2$ & $14.5 \pm 8.1$ & $16.8 \pm 8.0 *$ & $14.0 \pm 7.2 *$ \\
\hline$\geq \mathbf{3 0 . 0}(\%)$ & $6.2 \%$ & $8.6 \%$ & $3.4 \%$ & $11.1 \%$ & $2.6 \%$ \\
\hline $\mathbf{2 0 . 0}-\mathbf{2 9 . 9}(\%)$ & $14.7 \%$ & $14.3 \%$ & $15.3 \%$ & $14.8 \%$ & $14.7 \%$ \\
\hline$<\mathbf{2 0 . 0}(\%)$ & $79.1 \%$ & $77.1 \%$ & $81.3 \%$ & $74.1 \%$ & $82.7 \%$ \\
\hline
\end{tabular}

\section{Table 2. Serum 25(OH)D levels of the study population} $p<0.05$ mean $25(\mathrm{OH}) \mathrm{D}$ levels of COGHD patients vs. AOGHD patients

We also analyzed the distribution of the participants in the 3 categories of vitamin $D$ status (adequate, insufficient and deficient) but found no significant difference comparing men vs. Women $(p=0.115)$ and COGHD vs. AOGHD subjects $(p=0.239)$.

Mean 25(OH)D levels (15.1 $7.6 \mathrm{ng} / \mathrm{ml})$ corresponded to the high prevalence of impaired vitamin $D$ status in our cohort of GHD patients. 25(OH)D concentrations did not differ between men and women $(p=0.387$ ) (Table 2$)$ and were negatively and weakly correlated with age $(r=-0.256 ; p=0.003)$ (Data not shown). In the younger group of COGHD subjects, however, mean serum 25(OH)D was significantly higher compared with AOGHD participants $(p=0.039)($ Table 2$)$.

\section{Conclusion}

Data from our study demonstrated considerably high prevalence of hypovitaminosis D in GHD adults, with lower 25(OH)D concentrations among the subgroup with AOGHD. Therefore, 25(OH)D testing is highly recommended in patients with hyposomatotropism. Normalization of vitamin D status might have beneficial effects in GHD subjects, especially considering the additive effects of vitamin $\mathrm{D}$ and $\mathrm{GH}$ replacement.

\section{References:}$$
2 \text {. }
$$

like growth factor I concentrations in healthy subjects.

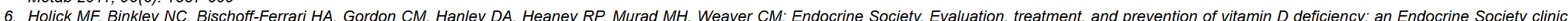
practice guideline. J. Clin. Endocrinol. Metab 2011; 96(7): 1911-30 